\title{
The Pathogenesis of Necroptosis-Dependent Signaling Pathway in Cerebral Ischemic Disease
}

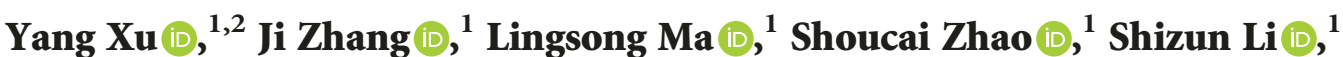 \\ Tingting Huang $\left(\mathbb{D},{ }^{1}\right.$ and Zhaohu Chu $\left(\mathbb{1}^{1}\right.$ \\ ${ }^{1}$ Department of Neurology, First Affiliated Hospital of Wannan Medical College, No. 2, ZheShanXi Road, Wuhu 241001, China \\ ${ }^{2}$ Non-Coding RNA of Major Diseases Research Center, Central Laboratory, First Affiliated Hospital of Wannan Medical College, \\ No. 2, ZheShanXi Road, Wuhu 241001, China
}

Correspondence should be addressed to Shizun Li; lishizun1212@163.com, Tingting Huang; 1006551570@qq.com, and Zhaohu Chu; chuzhaohu0991@163.com

Received 14 March 2018; Accepted 13 May 2018; Published 22 July 2018

Academic Editor: Hu Qin

Copyright (c) 2018 Yang Xu et al. This is an open access article distributed under the Creative Commons Attribution License, which permits unrestricted use, distribution, and reproduction in any medium, provided the original work is properly cited.

Necroptosis is the best-described form of regulated necrosis at present, which is widely recognized as a component of caspaseindependent cell death mediated by the concerted action of receptor-interacting protein kinase 1 (RIPK1) and receptorinteracting protein kinase 3 (RIPK3). Mixed-lineage kinase domain-like (MLKL) was phosphorylated by RIPK3 at the threonine 357 and serine 358 residues and then formed tetramers and translocated onto the plasma membrane, which destabilizes plasma membrane integrity leading to cell swelling and membrane rupture. Necroptosis is downstream of the tumor necrosis factor (TNF) receptor family, and also interaction with NOD-like receptor pyrin 3 (NLRP3) induced inflammasome activation. Multiple inhibitors of RIPK1 and MLKL have been developed to block the cascade of signal pathways for procedural necrosis and represent potential leads for drug development. In this review, we highlight recent progress in the study of roles for necroptosis in cerebral ischemic disease and discuss how these modifications delicately control necroptosis.

\section{Introduction}

For a long time, necrosis was classified as nonprogrammed cell death as a response to extreme stress. The integrity of the plasma membrane was attacked by uncontrolled and accidental necrosis causing the collapse of the cell, though the nuclei keep substantially intact on the way [1]. However, in recent years, there is strong evidence confirming that part of necrosis also contained program control, therefore proposing the new concept as programmed necrosis or necroptosis. Apoptosis, autophagy, and necroptosis are all classified as programmed cell death (PCD) based on morphological and biochemical features $[2,3]$. These phenomena have been observed in the ischemic stroke model [4-6]. Due to technical limitations, many studies considered necroptosis equated with apoptosis. Necroptosis is not induced by the caspase activation which is a typical requirement of the apoptotic pathway [7]. Wang et al. systematically exposed the classic signal pathway of necroptosis to further understand this form of cell death [8]. It was found that necroptotic cell death participates in a variety of cerebrovascular diseases. These mechanisms are reviewed in this paper, since they could be targets of new therapeutic approaches for these diseases.

\section{Research Progress of the Signal Pathway of Programmed Necrosis}

In the 1990s, researchers observed that caspase inhibition cannot fully block tumor necrosis factor- (TNF-) induced cell death but rather switches the cell fate to the necrotic death signal pathway similar to apoptosis $[9,10]$. This is the first time that procedural necroptosis has been observed. In the activation of downstream necroptosis of the pathway, caspase- 8 plays a critical regulatory role in the switch. Caspase- 8 acts as an endogenous inhibitor of the necroptosis 
signal. It not only cleaves but also activates and initiates the execution phase of apoptosis. When FADD-caspase-8-FLIP complex functions are inhibited, the pathway of cell death switches from apoptosis to typical necroptosis features [11-14]. On the other side, when RIP3 kinase activity is inhibited, necroptosis may also lead to the activation of the FADD-RIPK1-RIPK3-caspase-8 complex to induce apoptosis [15]. TNF- $\alpha$ is the major trigger of necroptosis, which is capable of initiating RIPK1 kinase-dependent necroptosis as well as caspase-8-dependent apoptosis [16].

So what is the most classic feature of procedural necroptosis? TNF- $\alpha$-induced necroptosis is mostly intensively investigated. TNF receptor 1 (TNFR1) ligation recruits complex I which contains TRADD, TRAF2, and cIAP1/2 [17]. Complex I transits into the cytosol activating deathinducing TNFR1 complex II via cylindromatosis (CYLD) [18]. In the necrotic signal pathway, receptor-interacting kinase 1 (RIPK1 or RIP1) was the first molecule recognized as the central components of the necroptotic machinery [19]. When FADD or caspase- 8 is inactivated or absent, RIPK1 and TRIF include a RIP homotypic interaction motif (RHIM) domain that permits RIPK3 activation via RHIMmediated interactions. RIPK3 kinase activity and RIPK3 RHIM domain are the requirement of necroptosis induction [20, 21]. RIPK1 and RIPK3 were phosphorylated, then formed a necrosome through their RHIM domains, and activate their kinase activities [22]. This RIPK1-RIPK3 interacts with mixed-lineage kinase domain-like (MLKL) phosphorylation [23], which consists of an N-terminal 4helical-bundle domain (4HBD) linked by a brace region to a C-terminal pseudokinase domain [24]. MLKL was phosphorylated by RIP3 at the serine 358 and threonine 357 residues, which induces a conformational change into its active state [25], and then formed tetramers and translocated onto the plasma membrane, which injures cellular membrane integrity resulting in cell swelling and membrane rupture [26]. Downstream of the necrosome are two splice variants of phosphoglycerate mutase family member 5 (PGAM5), PGAM5S and PGAM5L. It was first reported to be a key substrate associated with the RIP1-RIP3-MLKL complex in necroptosis. PGAM5S and PGAM5L are both requirements of intrinsic necroptosis. The presence of the necrosis inhibitor necrosulfonamide (NSA) does not affect PGAM5L bound to the necrosome. However, the binding of PGAM5S is blocked by NSA. PGAM5S is normally located on the mitochondria and becomes related to the upstream necrosis-inducing complex probably through interactions with RIP3 resulting in the activation of PGAM5S by phosphorylation [27]. Furthermore, mitochondrial fragmentation caused by the mitochondrial phosphatase PGAM5S which recruited the mitochondrial fission factor dynamin-related protein 1 (Drp1) may upregulate ROS generation [27].

RIP3 is a nucleocytoplasmic shuttling protein whose nuclear distribution is temperature-sensitive. RIP3 possesses two classical unconventional nuclear localization signals (NLS, aa 442-472) and two classical nuclear export signals (NES) [28]. The above two localization signals and amino acid residue 452 within NLS participated in the necroptosis signal pathway [29]. Therefore, some studies began to explore the existence of the RIP3 nuclear pathway in addition to the classic mitochondrial pathway. Recent literature suggests that RIP3 binds to the apoptosis-inducing factor (AIF) nuclear pathway which is also involved in the process of procedural necrosis [14]. AIF is discharged from mitochondria and combines with RIP3 to form RIP3-AIF complexes. The new RIP3-AIF complex translocates into the nucleus leading to DNA degradation, and then the neurons are induced to suffer the necroptosis signal pathway (Figure 1).

\section{Necroptosis in Cerebral Ischemia Disease}

In the field of cerebral ischemia, the recognition of procedural necrosis can be traced back to 2005; Degterev et al. found that cerebral ischemia in mice in the absence of apoptotic signaling is contributing to the nonapoptotic death pathway like necroptosis. Necroptosis delayed mouse ischemic brain injury distinct from that of apoptosis [30]. Vieira et al. established oxygen-glucose deprivation (OGD) models in vitro and explored the mechanism underlying OGDinduced necroptosis in hippocampal neurons, and they found that although ischemic insults induced caspase- 8 mRNA downregulation, they also induced RIP3 mRNA and protein level upregulation. The changes in RIP3 protein level were positively related to hippocampal neuronal death. Similar to RIP3, RIP1 protein levels were positively related to the activation of neuronal death. Both RIP1 and RIP3 contribute to necrotic cell death in hippocampal neurons challenged with OGD insult. Consistent with the classical procedural necroptosis cellular pathways, upregulation of RIP1-RIP3 expression and negative change of caspase- 8 can afterwards be used to activate necroptotic signaling [31].

Global brain ischemia and reperfusion (I/R) injury acts as another manifestation of brain cell injury, in which the hippocampal CA1 layer is especially vulnerable [32, 33]. Yin et al. built rat $20 \mathrm{~min}$ global cerebral ischemia model to explore intracellular changes [5]. As a marker of necroptosis, RIP3 upregulated and transferred into the nucleus after cerebral ischemia and reperfusion injury. The RIP1-RIP3 complex plays crucial roles for TNF-induced necroptosis in the cell cytosol. ATP depletion is one of the results of the mitochondrial permeability transition pore (mPTP) leading to mitochondrial swelling. Fakharnia et al. presented additional arguments in the classical mitochondrial pathway. As a gatekeeper of mPTP, CypD, which mediated mPTP opening, may contribute to not only apoptosis but also necroptotic cell death in cerebral I/R injury and alleviated the levels of RIP1 and RIP3 [34]. However, the RIP3 function in the nucleus is not completely dependent on the RIP3-RIP1 complex. It needs future studies to examine other nuclear proteins that interacted with RIP3 [5]. Xu et al. continued to explore the role of RIP3 mechanisms in the nucleus. The interaction between activated RIP3 and AIF occurs in the cytoplasm after I/R injury. AIF and RIP3 translocation into the nucleus is critical to neuronal necroptosis, and AIF translocation into the nucleus may be RIP3-dependent [14]. As a key mediator, AIF links caspase-independent PCD with the necroptotic pathway. 


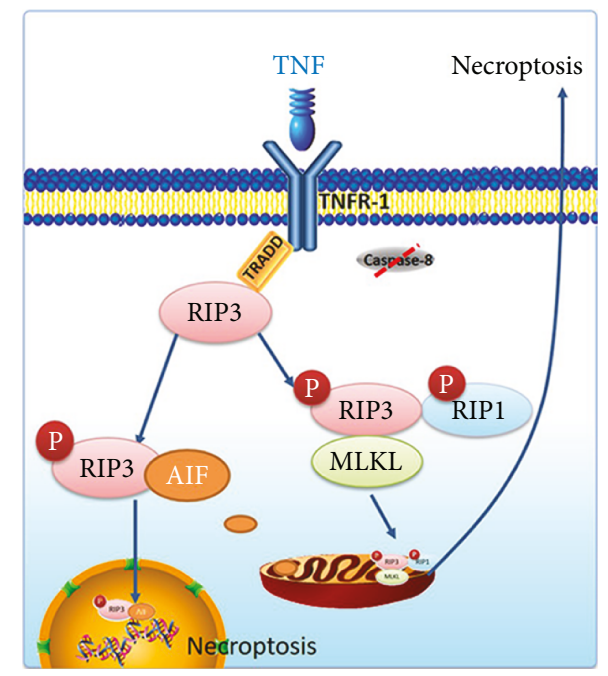

Figure 1: Two classic pathways of necroptosis. One of the classic pathways is RIP3 binding to RIP1 to form procedural necrosis complex II, which in turn binds to MLKL and mediates necroptosis of the mitochondrial pathway. The other is RIP1 binding to AIF, which translocated into the nucleus and mediates necroptosis of the nuclear pathway.

Nerve cell necrosis was observed following the focal middle carotid artery occlusion/reperfusion (MCAO/R) ischemic stroke model. TNFR1 and RIP3 were positively expressed and significantly increased following the volume of cerebral infarction postreperfusion. Caspase inhibitor z-VAD-FMK (z-VAD) treatment markedly increased RIP3 expression during ischemia injury [35]. Compared with the classic procedural necrosis pathway, $\mathrm{Lu}$ et al. put forward the opposite view on the MCAO model, which is mainly concentrated in the downstream of RIP1-RIP3-MLKL. They found the mitochondria-enriched RIP1/RIP3 complex in necroptosis; nevertheless, PGAM5 had hardly any effect on RIP1-RIP3 recruitment. PGAM5 gene knockout in mice exacerbated necroptosis rather than reduced necroptosis. It causes abnormal mitochondrial accumulation and increases ROS generation [36].

In addition to phosphorylation modification, Miao et al. tested RIP3 S-nitrosylation in I/R paralleled with elevated phosphorylation. It means that phosphorylation and activation of RIP3 could be modulated by its S-nitrosylation triggered by NMDAR-dependent nNOS activation [37].

\section{The Activation of the NLRP3 Inflammasome by a Mechanism Involving the RIP1-RIP3 Signaling Pathway}

Neuroinflammation is still the primary cause of morbidity and mortality in cerebral ischemia [38-40]. The NOD-like receptor pyrin 3 (NLRP3) inflammasome is considered an effective therapeutic target. The abnormalities of structure and expression of the NLRP3 inflammasome could affect the development or progression of ischemic stroke. After OGD, MCAO, or global cerebral ischemia injury, NLRP3

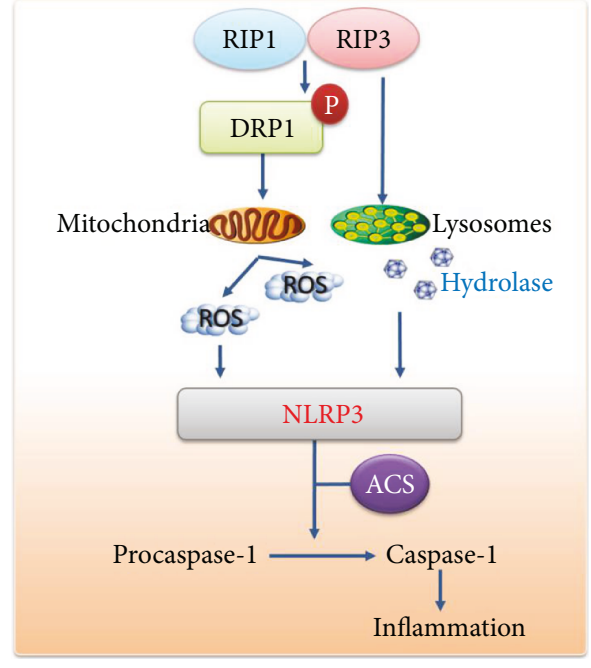

FIGURE 2: The activation of the NLRP3 inflammasome through the RIP1-RIP3 signaling pathway. The activation of RIP1-RIP3 damages the mitochondria by activating the DRP1. This results in excessive production of ROS and subsequent trigger activation of the NLRP3 inflammasome. RIP3 also destructs lysosomal membrane stability, leading to hydrolase release (such as cathepsin-B) and activation of NLRP3-mediated inflammatory factors.

inflammasome and other proinflammatory cytokines were activated [41]. These cytokines could be the mediating molecules during postischemic inflammation and immune responses. Following the detection of cellular stress, NLRP3 was exposed to interact with the adaptor apoptosisassociated speck-like protein containing a CARD (ASC). After binding with NLRP3, ASC recruits procaspase-1 clustering which permits autocleavage and formation of the active caspase-1, which mediates the release of the mature, biologically active cytokines to engage in immune defense [42]. The downstream of NLRP3 was explored relatively and clearly in neuronal cell experiments, primarily associated with inflammatory factors. Caspase- 1 and both IL- 6 and IL$1 \beta$ are involved in mouse primary cortical neurons' ischemic conditions $[43,44]$. In particular, caspase-1 inhibitor treatment protected neurons in experimental stroke models through suppression of NLRP3 inflammasome activity [45]. These mechanisms have been confirmed in the cerebral ischemic disease model. However, we know very little about its upstream studies. So far, the mechanism that activates NLRP3 inflammasome in ischemia injury generalizations in two main models, lysosomal damage or ROS release, is mutually connected and associated with NLRP3 in ischemia injury (Figure 2). Although there are many pathways of ROS production after ischemia [46, 47], necroptosis is one of the ways.

As mentioned before, caspase- 8 is a regulatory molecule. Kang found certain cells deficient in caspase- 8 prompting which is mediated by RIP1 and RIP3. Caspase- 8 deficiency dendritic cells (DCs) expressed to accentuated activation of the inflammasome through the functions of RIP1, RIP3, and MLKL [7]. And Gurung et al. considered caspase- 8 an apical mediator of NLRP3 inflammasome priming [48]. MLKL, a key component downstream of necrosome components, is 
considered an executor of necroptosis. In MLKL-knockout mice, NLRP3 activation was inhibited, which means that the potential function of MLKL is the regulation of inflammasome activation [17]. Beyond their core role in necrosis, the necrosome components RIP1 and RIP3 have been proposed to be hyperresponsive to the induction of assembly, which were applied to the NLRP3 inflammasome. When inhibitory factors of necrosomes are blocked, RIP1-RIP3 could promote inflammasome activation spontaneously. Wang et al. discovered that the RIP1-RIP3 complex participates in RNA virus-induced NLRP3 activation through the GTPase DRP1 pathway. This promoted the mitochondrial damage leading to the production of ROS and stimulus NLRP3 [49].

The relationship between the procedural necrosis and inflammation is likely clearer, but this may not be the only signaling pathways that intersect. At least downstream studies, including the relationship between necroptosis and inflammation in cerebral infarction, indicate the direction of the study.

\section{The Regulation of Necroptosis in the Cerebral Ischemic Model}

Many studies focus on how to block the cascade of signal pathways for procedural necrosis. The most classic inhibitor is the small-molecule compound necrosulfonamide (NSA). NSA did not block interactions between necrosis-induced RIP1 and RIP3, but it blocks necroptosis downstream of RIP3 activation. NSA targets the N-terminal Cys86 residue MLKL which has a drawback that is specifically inhibiting necroptosis in multiple human cell lines. In human glioblastoma cells, NSA switches from necrosis to apoptosis in edelfosine-treated cells [50]. In a HeLa cell line in which caspase-8 was knocked down and RIP3 was expressed, necrosis induced by TNF- $\alpha$ plus Smac mimetic (no need for z-VAD) was efficiently blocked by NSA, while necrosis induced by either TNF- $\alpha$ or z-VAD was insensitive to NSA in a 3 T3 cell line expressing mouse RIP3 [23]. NSA also significantly reduces BV6/MS275-induced cell death in acute myeloid leukemia (AML) cell lines [51].

In the field of cerebral ischemia, some of the inhibitors of programmed necrosis have been demonstrated in animal models. Degterev et al. reported that as an identified smallmolecule inhibitor of necroptosis, necrostatin-1 (NEC-1) has been shown to ameliorate tissue damage in ischemic brain injury animal models [30]. NEC-1 has a selective primary cellular target responsible for the death domain receptor-associated adaptor kinase RIP1 activity [52, 53]. The crystal structures of the RIP1 kinase domain bound to NEC-1 and are caged in a hydrophobic pocket between the $\mathrm{N}$ - and C-lobes of the kinase domain. This structure stabilizes RIP1 in an inactive conformation. DAXX is a novel substrate of RIP3 in global cerebral ischemia and ischemia of the retinal cell animal model and is translocated from the nucleus to the cytoplasm in response to stress. Pretreatment with Nec-1 can block DAXX translocation from the nucleus to the cytoplasm, which resulted in the inactivation of DAXX [54-56]. NEC-1 not only inhibited the expression of RIP1 and prevented the upregulation and nuclear translocation of RIP3 but also decreased cathepsin-B release in the globe cerebral ischemic model. This suggests that there may be a signal transduction between programmed necrosis and autophagy [5]. The recent literature highlights the intricate interplay between necroptosis and autophagy. CA074-me and 3-methyladenine (3-MA), as autophagy inhibitors [46], were used to determine what is beneficial for global cerebral ischemia in the process of necroptosis signal pathways. CA074-me and 3-MA pretreatment greatly inhibited rat mortality rates and neuronal death. The mechanism of 3MA is the inhibition of the nuclear translocation and colocalization of RIP3 and AIF, as it is significant for ischemic neuronal DNA degradation and necroptosis for the nuclear translocation of the RIP3-AIF complex [14]. In the stabilization of the lysosomal membrane, CA074-me has an indirect effect by maintaining energy balance and inhibiting RIP3 expression and nuclear translocation [57]. Besides this, CA074-me almost completely hampered the loss of mitochondrial membrane depolarization, phosphatidylserine (PS) translocation, and plasma membrane rupture [58].

Multitargeted kinase inhibitors, such as dabrafenib, vemurafenib, sorafenib, pazopanib, and ponatinib, are currently used for the treatment of cancer, which have later emerged as having antinecroptotic activity [59-61]. Among them, dabrafenib may block TNF- $\alpha$-induced necroptosis as an effective high-affinity inhibitor of RIP3. Dabrafenib administered intraperitoneally after mouse cerebral ischemic injury markedly reduced infarct lesion size along with significantly attenuated upregulation of TNF- $\alpha$ [59].

\section{Future Research about Necroptosis}

Previous studies suggested that PGAM5 promotes necroptosis by associating with necrosome. However, the role of PGAM5 intrinsically is still controversial [62]. In response to multiple necroptotic stimuli, PGAM5 deficiency aggravated rather than mitigated necroptosis in brain ischemic reperfusion injury [36]. The loss of PGAM caused abnormal mitochondrial accumulation and increased ROS generation. In oxidative damage and necroptosis-dependent stroke, PGAM5 could drive pathology, and thus targeting PGAM5 may be of benefit.

The core of procedural necrosis is RIP1-RIP3 and its downstream signaling pathways. In addition to the abovementioned inhibitors, the drugs used in the experiments and the clinical use of drugs have been developed. This is because the understanding of this pathway is still relatively limited. After the classic pathway was proposed, there are many studies that questioned the effect of RIP3 on the necrosis of the bypass signals, such as dynamin-related protein 1 and proteasome beta- 4 subunit (PSMB4) $[63,64]$. These need to be systematically assessed. Furthermore, the most critical problem is that for a long time, both necrotic and apoptotic cells dominated the theory of neuronal death in the penumbra zone. The clarification of the relationship between procedural necroptosis and necrosis and autophagy still needs a lot of work to do. This cross-channel signal path may be complexity over imagination. 


\section{Conflicts of Interest}

The authors declare that they have no conflicts of interest.

\section{Acknowledgments}

This work was supported by the National Natural Science Foundation of China (Grant nos. 81701161 and 81371295).

\section{References}

[1] D. V. Krysko, T. vanden Berghe, K. D'Herde, and P. Vandenabeele, "Apoptosis and necrosis: detection, discrimination and phagocytosis," Methods, vol. 44, no. 3, pp. 205221, 2008.

[2] Y. Fuchs and H. Steller, "Programmed cell death in animal development and disease," Cell, vol. 147, no. 4, pp. 742-758, 2011.

[3] Y. Fuchs and H. Steller, "Live to die another way: modes of programmed cell death and the signals emanating from dying cells," Nature Reviews Molecular Cell Biology, vol. 16, no. 6, pp. 329-344, 2015.

[4] B. Pourheydar, S. Soleimani Asl, M. Azimzadeh, A. Rezaei Moghadam, A. Marzban, and M. Mehdizadeh, "Neuroprotective effects of bone marrow mesenchymal stem cells on bilateral common carotid arteries occlusion model of cerebral ischemia in rat," Behavioural Neurology, vol. 2016, Article ID 2964712, 10 pages, 2016.

[5] B. Yin, Y. Xu, R. L. Wei, F. He, B. Y. Luo, and J. Y. Wang, "Inhibition of receptor-interacting protein 3 upregulation and nuclear translocation involved in necrostatin-1 protection against hippocampal neuronal programmed necrosis induced by ischemia/reperfusion injury," Brain Research, vol. 1609, pp. 63-71, 2015.

[6] H. Wei, Y. Li, S. Han et al., "cPKC $\gamma$-modulated autophagy in neurons alleviates ischemic injury in brain of mice with ischemic stroke through Akt-mTOR pathway," Translational Stroke Research, vol. 7, no. 6, pp. 497-511, 2016.

[7] A. Kaczmarek, P. Vandenabeele, and D. V. Krysko, "Necroptosis: the release of damage-associated molecular patterns and its physiological relevance," Immunity, vol. 38, no. 2, pp. 209223, 2013.

[8] S. He, L. Wang, L. Miao et al., "Receptor interacting protein kinase-3 determines cellular necrotic response to TNF-alpha," Cell, vol. 137, no. 6, pp. 1100-1111, 2009.

[9] A. Kawahara, Y. Ohsawa, H. Matsumura, Y. Uchiyama, and S. Nagata, "Caspase-independent cell killing by Fas-associated protein with death domain," The Journal of Cell Biology, vol. 143, no. 5, pp. 1353-1360, 1998.

[10] D. Vercammen, R. Beyaert, G. Denecker et al., "Inhibition of caspases increases the sensitivity of L929 cells to necrosis mediated by tumor necrosis factor," The Journal of Experimental Medicine, vol. 187, no. 9, pp. 1477-1485, 1998.

[11] C. Günther, E. Martini, N. Wittkopf et al., "Caspase-8 regulates TNF- $\alpha$-induced epithelial necroptosis and terminal ileitis," Nature, vol. 477, no. 7364, pp. 335-339, 2011.

[12] W. J. Kaiser, J. W. Upton, A. B. Long et al., "RIP3 mediates the embryonic lethality of caspase-8-deficient mice," Nature, vol. 471, no. 7338, pp. 368-372, 2011.

[13] A. Oberst, C. P. Dillon, R. Weinlich et al., "Catalytic activity of the caspase-8-FLIP(L) complex inhibits RIPK3-dependent necrosis," Nature, vol. 471, no. 7338, pp. 363-367, 2011.
[14] Y. Xu, J. Wang, X. Song et al., "RIP3 induces ischemic neuronal DNA degradation and programmed necrosis in rat via AIF," Scientific Reports, vol. 6, no. 1, p. 29362, 2016.

[15] I. Shlomovitz, S. Zargrian, and M. Gerlic, "Mechanisms of RIPK3-induced inflammation," Immunology and Cell Biology, vol. 95, no. 2, pp. 166-172, 2017.

[16] P. Vandenabeele, L. Galluzzi, T. vanden Berghe, and G. Kroemer, "Molecular mechanisms of necroptosis: an ordered cellular explosion," Nature Reviews Molecular Cell Biology, vol. 11, no. 10, pp. 700-714, 2010.

[17] X. Zhang, C. Fan, H. Zhang et al., "MLKL and FADD are critical for suppressing progressive lymphoproliferative disease and activating the NLRP3 inflammasome," Cell Reports, vol. 16, no. 12, pp. 3247-3259, 2016.

[18] J. Hitomi, D. E. Christofferson, A. Ng et al., "Identification of a molecular signaling network that regulates a cellular necrotic cell death pathway," Cell, vol. 135, no. 7, pp. 13111323, 2008.

[19] N. Holler, R. Zaru, O. Micheau et al., "Fas triggers an alternative, caspase-8-independent cell death pathway using the kinase RIP as effector molecule," Nature Immunology, vol. 1, no. 6, pp. 489-495, 2000.

[20] Y. S. Cho, S. Challa, D. Moquin et al., "Phosphorylation-driven assembly of the RIP1-RIP3 complex regulates programmed necrosis and virus-induced inflammation," Cell, vol. 137, no. 6, pp. 1112-1123, 2009.

[21] J. W. Upton, W. J. Kaiser, and E. S. Mocarski, "Virus inhibition of RIP3-dependent necrosis," Cell Host \& Microbe, vol. 7, no. 4, pp. 302-313, 2010.

[22] J. Li, T. McQuade, A. B. Siemer et al., "The RIP1/RIP3 necrosome forms a functional amyloid signaling complex required for programmed necrosis," Cell, vol. 150, no. 2, pp. 339-350, 2012.

[23] L. Sun, H. Wang, Z. Wang et al., "Mixed lineage kinase domain-like protein mediates necrosis signaling downstream of RIP3 kinase," Cell, vol. 148, no. 1-2, pp. 213-227, 2012.

[24] J. M. Murphy, P. E. Czabotar, J. M. Hildebrand et al., "The pseudokinase MLKL mediates necroptosis via a molecular switch mechanism," Immunity, vol. 39, no. 3, pp. 443-453, 2013.

[25] D. A. Rodriguez, R. Weinlich, S. Brown et al., "Characterization of RIPK3-mediated phosphorylation of the activation loop of MLKL during necroptosis," Cell Death and Differentiation, vol. 23, no. 1, pp. 76-88, 2016.

[26] Z. Cai, S. Jitkaew, J. Zhao et al., "Plasma membrane translocation of trimerized MLKL protein is required for TNF-induced necroptosis," Nature Cell Biology, vol. 16, no. 1, pp. 55-65, 2014.

[27] Z. Wang, H. Jiang, S. Chen, F. du, and X. Wang, "The mitochondrial phosphatase PGAM5 functions at the convergence point of multiple necrotic death pathways," Cell, vol. 148, no. 1-2, pp. 228-243, 2012.

[28] Y. Yang, J. Ma, Y. Chen, and M. Wu, "Nucleocytoplasmic shuttling of receptor-interacting protein 3 (RIP3): identification of novel nuclear export and import signals in RIP3," The Journal of Biological Chemistry, vol. 279, no. 37, pp. 3882038829, 2004.

[29] M. Li, S. Feng, and M. Wu, "Multiple roles for nuclear localization signal (NLS, aa 442-472) of receptor interacting protein 3 (RIP3)," Biochemical and Biophysical Research Communications, vol. 372, no. 4, pp. 850-855, 2008. 
[30] A. Degterev, Z. Huang, M. Boyce et al., "Chemical inhibitor of nonapoptotic cell death with therapeutic potential for ischemic brain injury," Nature Chemical Biology, vol. 1, no. 2, pp. 112119, 2005.

[31] M. Vieira, J. Fernandes, L. Carreto et al., "Ischemic insults induce necroptotic cell death in hippocampal neurons through the up-regulation of endogenous RIP3," Neurobiology of Disease, vol. 68, pp. 26-36, 2014.

[32] K. R. Dave, D. Della-Morte, I. Saul, R. Prado, and M. A. PerezPinzon, "Ventricular fibrillation-induced cardiac arrest in the rat as a model of global cerebral ischemia," Translational Stroke Research, vol. 4, no. 5, pp. 571-578, 2013.

[33] J. J. Szymanski, H. Wang, J. T. Jamison, and D. J. DeGracia, "HuR function and translational state analysis following global brain ischemia and reperfusion," Translational Stroke Research, vol. 4, no. 6, pp. 589-603, 2013.

[34] F. Fakharnia, F. Khodagholi, L. Dargahi, and A. Ahmadiani, "Prevention of cyclophilin D-mediated mPTP opening using cyclosporine-a alleviates the elevation of necroptosis, autophagy and apoptosis-related markers following global cerebral ischemia-reperfusion," Journal of Molecular Neuroscience, vol. 61, no. 1, pp. 52-60, 2017.

[35] Y. Dong, C. Bao, J. Yu, and X. Liu, "Receptor-interacting protein kinase 3-mediated programmed cell necrosis in rats subjected to focal cerebral ischemia-reperfusion injury," Molecular Medicine Reports, vol. 14, no. 1, pp. 728-736, 2016.

[36] W. Lu, J. Sun, J. S. Yoon et al., "Mitochondrial protein PGAM5 regulates mitophagic protection against cell necroptosis," PLoS One, vol. 11, no. 1, article e0147792, 2016.

[37] W. Miao, Z. Qu, K. Shi et al., "RIP3 S-nitrosylation contributes to cerebral ischemic neuronal injury," Brain Research, vol. 1627, pp. 165-176, 2015.

[38] M. Ahmad and S. H. Graham, "Inflammation after stroke: mechanisms and therapeutic approaches," Translational Stroke Research, vol. 1, no. 2, pp. 74-84, 2010.

[39] J. Montaner, D. Salat, T. García-Berrocoso et al., "Reperfusion therapy for acute stroke improves outcome by decreasing neuroinflammation," Translational Stroke Research, vol. 1, no. 4, pp. 261-267, 2010.

[40] J. C. Pena-Philippides, Y. Yang, O. Bragina, S. Hagberg, E. Nemoto, and T. Roitbak, "Effect of pulsed electromagnetic field (PEMF) on infarct size and inflammation after cerebral ischemia in mice," Translational Stroke Research, vol. 5, no. 4, pp. 491-500, 2014.

[41] S. K. McCann, F. Cramond, M. R. Macleod, and E. S. Sena, "Systematic review and meta-analysis of the efficacy of interleukin-1 receptor antagonist in animal models of stroke: an update," Translational Stroke Research, vol. 7, no. 5, pp. 395-406, 2016.

[42] W. L. Lu, D. Z. Song, J. L. Yue et al., "NLRP3 inflammasome may regulate inflammatory response of human periodontal ligament fibroblasts in an apoptosis-associated speck-like protein containing a CARD (ASC)-dependent manner," International Endodontic Journal, vol. 50, no. 10, pp. 967-975, 2017.

[43] J. A. Switzer, A. Sikora, A. Ergul, J. L. Waller, D. C. Hess, and S. C. Fagan, "Minocycline prevents IL-6 increase after acute ischemic stroke," Translational Stroke Research, vol. 3, no. 3, pp. 363-368, 2012.

[44] C. P. Wang, L. Z. Zhang, G. C. Li et al., "Mulberroside A protects against ischemic impairment in primary culture of rat cortical neurons after oxygen-glucose deprivation followed by reperfusion," Journal of Neuroscience Research, vol. 92, no. 7, pp. 944-954, 2014.

[45] J. Qiu, M. Wang, J. Zhang et al., "The neuroprotection of sinomenine against ischemic stroke in mice by suppressing NLRP3 inflammasome via AMPK signaling," International Immunopharmacology, vol. 40, pp. 492-500, 2016.

[46] H. Li, A. Gao, D. Feng et al., "Evaluation of the protective potential of brain microvascular endothelial cell autophagy on blood-brain barrier integrity during experimental cerebral ischemia-reperfusion injury," Translational Stroke Research, vol. 5, no. 5, pp. 618-626, 2014.

[47] L. J. Wu, "Microglial voltage-gated proton channel Hv1 in ischemic stroke," Translational Stroke Research, vol. 5, no. 1, pp. 99-108, 2014.

[48] P. Gurung, P. K. Anand, R. K. S. Malireddi et al., "FADD and caspase- 8 mediate priming and activation of the canonical and noncanonical Nlrp3 inflammasomes," The Journal of Immunology, vol. 192, no. 4, pp. 1835-1846, 2014.

[49] X. Wang, W. Jiang, Y. Yan et al., "RNA viruses promote activation of the NLRP3 inflammasome through a RIP1-RIP3DRP1 signaling pathway," Nature Immunology, vol. 15, no. 12, pp. 1126-1133, 2014.

[50] S. Melo-Lima, M. C. Lopes, and F. Mollinedo, "Necroptosis is associated with low procaspase- 8 and active RIPK1 and -3 in human glioma cells," Oncoscience, vol. 1, no. 10, pp. 649664, 2014.

[51] S. Steinwascher, A. L. Nugues, H. Schoeneberger, and S. Fulda, "Identification of a novel synergistic induction of cell death by Smac mimetic and HDAC inhibitors in acute myeloid leukemia cells," Cancer Letters, vol. 366, no. 1, pp. 32-43, 2015.

[52] A. Degterev, J. Hitomi, M. Germscheid et al., "Identification of RIP1 kinase as a specific cellular target of necrostatins," Nature Chemical Biology, vol. 4, no. 5, pp. 313-321, 2008.

[53] P. Vandenabeele, W. Declercq, and T. V. Berghe, "Necrotic cell death and 'necrostatins': now we can control cellular explosion," Trends in Biochemical Sciences, vol. 33, no. 8, pp. 352355, 2008.

[54] T. Xie, W. Peng, Y. Liu et al., "Structural basis of RIP1 inhibition by necrostatins," Structure, vol. 21, no. 3, pp. 493-499, 2013.

[55] R. Yang, K. Hu, J. Chen et al., "Necrostatin-1 protects hippocampal neurons against ischemia/reperfusion injury via the RIP3/DAXX signaling pathway in rats," Neuroscience Letters, vol. 651, pp. 207-215, 2017.

[56] Y. S. Lee, Y. Dayma, M. Y. Park, K. I. Kim, S. E. Yoo, and E. Kim, "Daxx is a key downstream component of receptor interacting protein kinase 3 mediating retinal ischemic cell death," FEBS Letters, vol. 587, no. 3, pp. 266-271, 2013.

[57] Y. Xu, J. Wang, X. Song et al., "Protective mechanisms of CA074-me (other than cathepsin-B inhibition) against programmed necrosis induced by global cerebral ischemia/reperfusion injury in rats," Brain Research Bulletin, vol. 120, pp. 97-105, 2016.

[58] Z. A. Dunai, G. Imre, G. Barna et al., "Staurosporine induces necroptotic cell death under caspase-compromised conditions in U937 cells," PLoS One, vol. 7, no. 7, article e41945, 2012.

[59] S. A. Cruz, Z. Qin, A. F. R. Stewart, and H. H. Chen, "Dabrafenib, an inhibitor of RIP3 kinase-dependent necroptosis, reduces ischemic brain injury," Neural Regeneration Research, vol. 13, no. 2, pp. 252-256, 2018. 
[60] P. Geserick, J. Wang, R. Schilling et al., "Absence of RIPK3 predicts necroptosis resistance in malignant melanoma," Cell Death \& Disease, vol. 6, no. 9, p. e1884, 2015.

[61] F. Feldmann, B. Schenk, S. Martens, P. Vandenabeele, and S. Fulda, "Sorafenib inhibits therapeutic induction of necroptosis in acute leukemia cells," Oncotarget, vol. 8, no. 40, pp. 68208-68220, 2017.

[62] W. Xu, L. Jing, Q. Wang et al., "Bax-PGAM5L-Drp1 complex is required for intrinsic apoptosis execution," Oncotarget, vol. 6, no. 30, pp. 30017-30034, 2015.

[63] C. Wu, J. Chen, Y. Liu et al., "Upregulation of PSMB4 is associated with the necroptosis after spinal cord injury," Neurochemical Research, vol. 41, no. 11, pp. 3103-3112, 2016.

[64] T. Yamada, Y. Adachi, M. Fukaya, M. Iijima, and H. Sesaki, "Dynamin-related protein 1 deficiency leads to receptorinteracting protein kinase 3-mediated necroptotic neurodegeneration," The American Journal of Pathology, vol. 186, no. 11, pp. 2798-2802, 2016. 


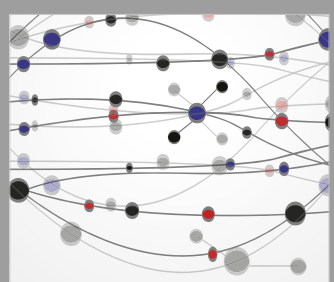

The Scientific World Journal
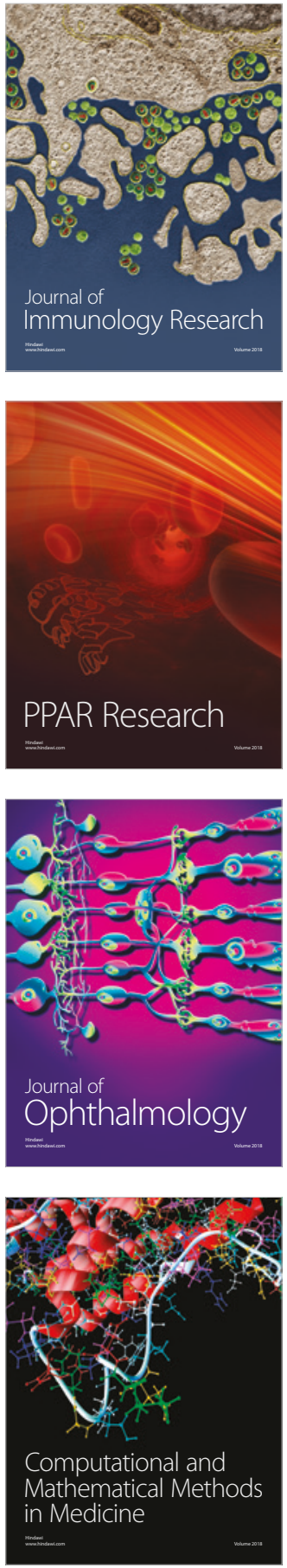

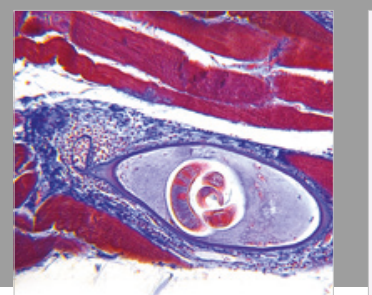

Gastroenterology Research and Practice

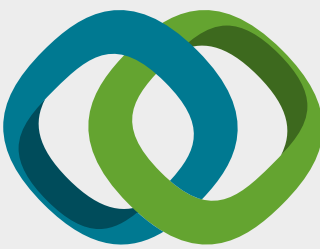

\section{Hindawi}

Submit your manuscripts at

www.hindawi.com
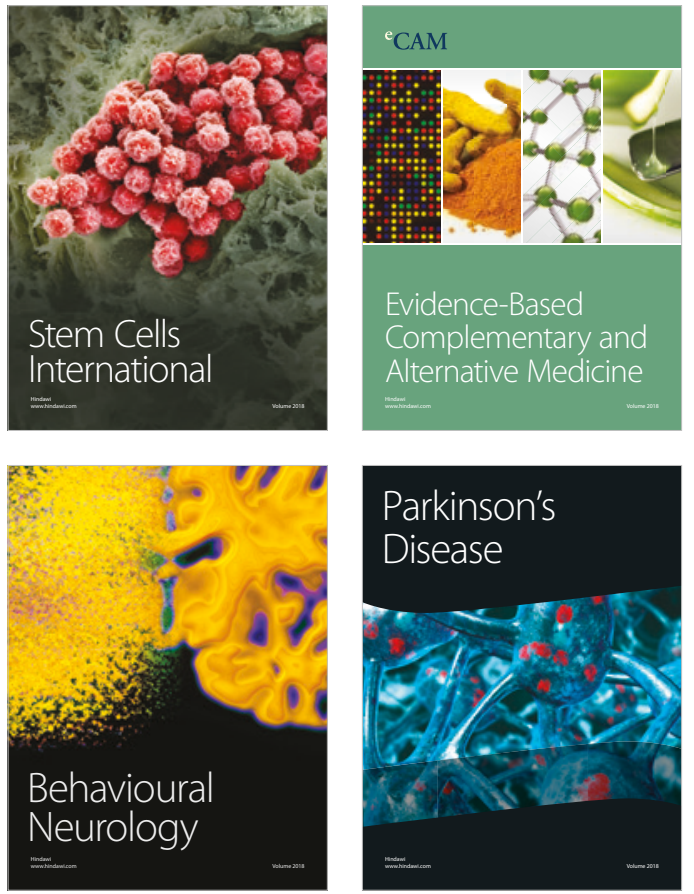

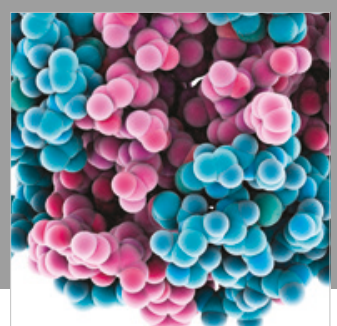

ournal of

Diabetes Research

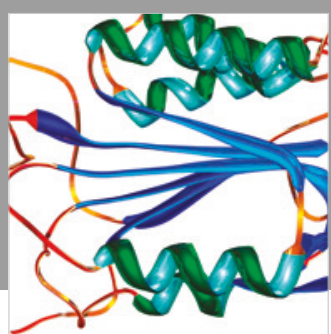

Disease Markers
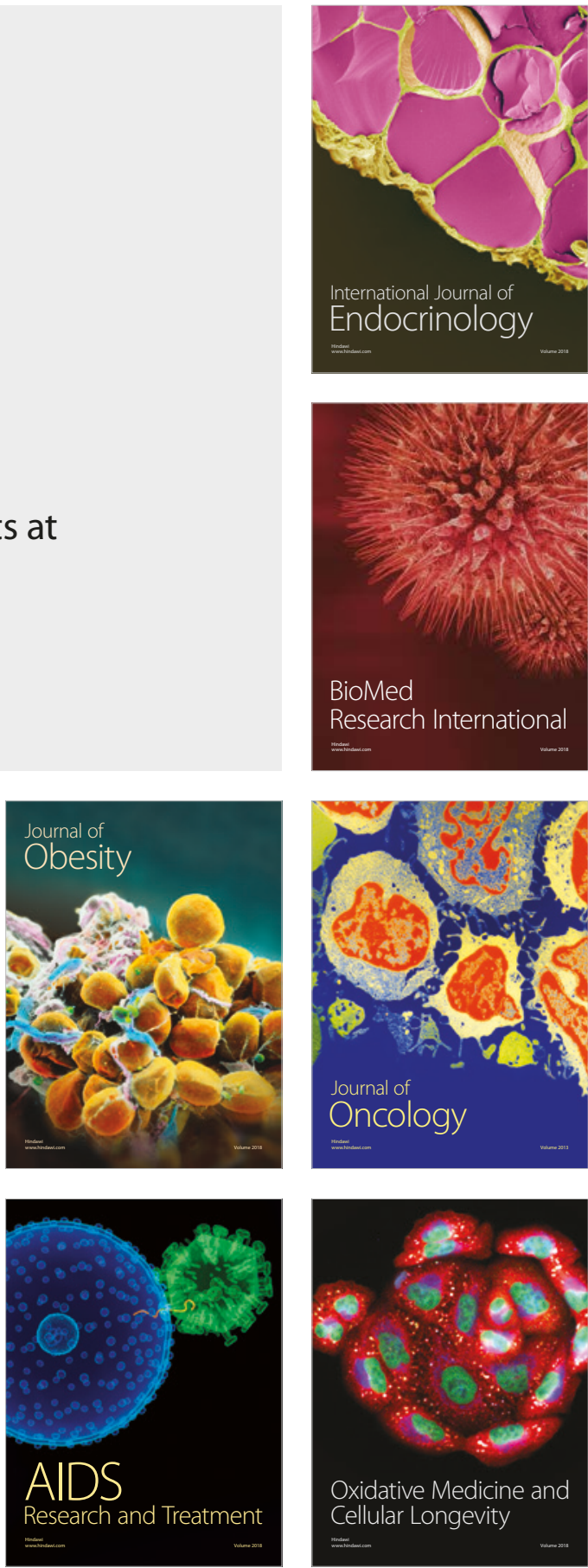